\title{
Estimação de volume de Pterogyne nitens em plantio puro no sudoeste da Bahia
}

\author{
Magno Pacheco Fraga ${ }^{1}$, Patrícia Anjos Bittencourt Barreto ${ }^{1}$, Alessandro de Paula ${ }^{1}$ \\ ${ }^{1}$ Universidade Estadual do Sudoeste da Bahia, Estrada do Bem Querer, Km 4, CEP 45083-900, Vitória da Conquista, BA, Brasil
}

"Autor correspondente:
magnofraga@hotmail.com

Termos para indexação:

Modelos volumétricos

Fator de forma

Quociente de forma

Amendoim-bravo

\section{Index terms:}

Volumetric models

Form factor

Form quotient

Histórico do artigo:

Recebido em 06/05/2014

Aprovado em 19/09/2014

Publicado em 10/10/2014

doi: 10.4336/2014.pfb.34.79.703
Resumo - A obtenção do volume é fundamental para o entendimento do potencial produtivo de um povoamento florestal. No entanto, como esta variável não é facilmente mensurável, torna-se necessária a obtenção de estimativas. Esse estudo teve como objetivos selecionar modelos volumétricos e avaliar a eficiência de três diferentes métodos para estimar o volume de madeira (fator de forma, quociente de forma e emprego de equações volumétricas ajustadas) para a espécie Pterogyne nitens Tul., em plantio puro no Município de Vitória da Conquista, BA, Brasil. Foram cortadas e cubadas, rigorosamente, árvores para obtenção do volume individual. Foram ajustados oito modelos volumétricos, sendo os melhores modelos selecionados com base no critério de seleção do valor ponderado dos escores dos parâmetros estatísticos e distribuição de resíduos. O modelo de Stoate apresentou melhor performance para estimar tanto o volume do fuste quanto o volume total da árvore com casca de Pterogyne nitens. Todavia, para o volume do fuste, o modelo de Koperzky-Gehrhardt também proporcionou estimativas semelhantes ao anterior, podendo ser também indicado para a espécie. Dentre os métodos utilizados para estimar o volume, o mais recomendado é o emprego de modelos volumétricos ajustados.

\section{Volume estimation of Pterogyne nitens in pure plantation in the southwest of Bahia}

\begin{abstract}
The knowledge of wood volume is essential to determine the logging productive potential of a forest plantation. However, as this variable isn't easily measurable it's necessary the obtainment by estimation. This study aims to select volumetric models and analyze the efficiency of three different methods to estimate the wood volume (form factor, form quotient and adjusted volumetric equation) to the species Pterogyne nitens Tul. in pure plantation in Vitoria da Conquista, State of Bahia, Brazil. The sampled trees were logged and rigorously cubed, andeight volumetric models were adjusted. The best models were selected based on the pondered value of the statistical parameters scores and residues distribution. Stoate model presented the best performance to estimate the bole volume and the total wood volume of Pterogyne nitens with bark. Nevertheless, for the bole volume, Koperzky and Gehrhardt model presented similar estimates as Stoate's, being also indicated to this species. Among the methods used to estimate volume, it is recommended the use of adjusted volumetric models.
\end{abstract}




\section{Introdução}

Em consequência da elevada demanda energética vegetal na Bahia, a lenha e o carvão ainda constituem os recursos florestais mais requeridos e utilizados, representando cerca de $30 \%$ da energia primária da região (Vasconcelos, 2012). No sudoeste do estado, apesar da maior parte desses produtos florestais serem oriundos de remanescentes de matas nativas, principalmente da caatinga, o progressivo aumento das áreas plantadas com espécies do gênero Eucalyptus vem contribuindo para o suprimento de produtos florestais.

A utilização de espécies florestais nativas em plantios homogêneos também pode representar uma importante alternativa para o fornecimento de produtos florestais. No entanto, como a escolha de espécies deve considerar não apenas a aptidão em relação ao sítio, mas também a sua produtividade (Lamprecht, 2000), as espécies nativas muitas vezes não são utilizadas por não se conseguir despertar interesse equivalente, na maioria das vezes, pela inexistência de informações relativas à sua ecologia, silvicultura e biometria (Tonini et al., 2005). De fato, ainda hoje são escassos os estudos relacionados a povoamentos homogêneos dessas espécies, o que limita o entendimento do seu potencial produtivo e utilização adequada.

A estimativa do potencial disponível em um povoamento florestal é normalmente obtida com base no volume madeireiro ou na biomassa produzida, por meio de inventário florestal que utiliza o conhecimento de variáveis de uma amostra para inferir sobre os parâmetros da população. Como o volume não é facilmente mensurável, vários métodos são utilizados para a sua estimativa. Segundo Soares et al. (2006), os mais comumente utilizados são o fator de forma, o quociente de forma e modelos volumétricos ajustados a partir de dados de cubagem rigorosa.

De acordo com Campos \& Leite (2006), o emprego de modelos volumétricos constitui o procedimento mais eficiente para a quantificação da produção em volume de um povoamento florestal. Muitas equações matemáticas foram propostas para estimar o volume de árvores e, apesar da eficiência de alguns modelos, estes nem sempre se adéquam a todas as espécies e condições, tornando necessário o ajuste de equações.

No Brasil, existe um grande número de estudos analisando diferentes métodos de estimação do volume para espécies florestais exóticas, principalmente para os gêneros Eucalyptus e Pinus em diferentes regiões do país (Machado et al., 2005; Oliveira et al., 2009; Soares et al., 2010; Almeida et al., 2011; Azevedo et al., 2011; Pelissari et al., 2011; Melo et al., 2013). No entanto, ainda são restritos os estudos voltados para obtenção de variáveis dendrométricas de espécies florestais nativas em plantios homogêneos (Araújo et al., 2004; Tonini et al., 2005), o que evidencia a importância de estudos relacionados ao tema.

Dentre as espécies nativas que apresentam potencial para utilização no Estado da Bahia, a madeira-nova ou também conhecida como amendoim-bravo (Pterogyne nitens Tul) destaca-se por se adaptar às condições ambientais da região, apresentar rápido crescimento e gerar madeira de valor econômico para diferentes finalidades, como para lenha, apresentando boa qualidade devido ao seu poder calorífico (Carvalho, 1994) e na construção de móveis (Lorenzi, 2002). Além disso, a espécie apresenta facilidade de estabelecimento em solos pobres em nutrientes e matéria orgânica, como consequência da capacidade de fixar nitrogênio pela simbiose com bactérias diazotróficas e fungos micorrízicos (Franco et al., 1995).

Portanto, este trabalho teve como objetivos selecionar modelos volumétricos e avaliar a eficiência de diferentes métodos para estimar o volume de madeira de Pterogyne nitens Tul. em plantio puro no sudoeste da Bahia.

\section{Material e métodos}

\section{Localização e caracterização da área}

O estudo foi realizado em um plantio puro de Pterogyne nitens, com cerca de seis anos de idade e espaçamento inicial de $3 \mathrm{~m} \times 3 \mathrm{~m}$. O plantio apresenta área de 0,72 hectare e está localizado no Campo Agropecuário da Universidade Estadual do Sudoeste da Bahia, Vitória da Conquista, BA.

A região apresenta temperatura média anual de $21^{\circ} \mathrm{C}$ e precipitação variando entre $700 \mathrm{~mm}$ e $1.100 \mathrm{~mm}$ anuais, distribuída nos meses de novembro a março, com um período seco de quatro a cinco meses. O clima da região é o do tipo Cwb segundo a classificação de Köppen, clima tropical de altitude (Sousa et al., 2013). O relevo é plano a levemente ondulado, com altitude em torno de $880 \mathrm{~m}$. O solo pertence à classe Latossolo Amarelo Distrófico (Santos et al., 2006). A vegetação predominante na região é classificada como Floresta Estacional Semidecidual Montana (Veloso et al., 1991). 


\section{Inventário florestal expedito e seleção das árvores}

Com propósito de obter medidas de altura total e diâmetro à altura de 1,3 m (DAP) de todos os indivíduos da população, foi realizado um inventário em $100 \%$ do povoamento.

As medidas de DAP dos fustes foram agrupadas em classes de diâmetro com amplitude correspondendo ao desvio padrão das medidas obtidas $(2,1 \mathrm{~cm})$, conforme proposto por Scolforo \& Figueiredo Filho (1998). Foram selecionadas e cortadas 30 árvores, que continham de um a cinco fustes, distribuídos nas diferentes classes de diâmetro (Tabela 1), totalizando 80 fustes medidos, com DAP mínimo de $1,8 \mathrm{~cm}$, máximo de $12,2 \mathrm{~cm}$ e altura mínima de 2,5 m e máxima de $10,5 \mathrm{~m}$. O número de árvores amostradas foi definido com base em estudos da literatura relacionada, que adotaram número amostral entre 10 e 40 árvores (Rios \& Mori, 2002; Ferrari et al., 2005; Moraes Neto, 2009; Soares et al., 2010; Azevedo et al., 2011; Melo et al., 2013).

Tabela 1. Distribuição diamétrica dos fustes de árvores de Pterogyne nitens em povoamento puro no sudoeste da Bahia.

\begin{tabular}{cccc}
\hline $\begin{array}{c}\text { Classes de } \\
\text { diâmetro } \\
\text { (cm) }\end{array}$ & $\begin{array}{c}\text { Centro de } \\
\text { classe } \\
\text { (cm) }\end{array}$ & $\begin{array}{c}\text { Frequência } \\
\text { de fustes }\end{array}$ & $\begin{array}{c}\text { Número } \\
\text { de fustes } \\
\text { selecionados }\end{array}$ \\
\hline $1,0-3,09$ & 2,0 & 92 & 14 \\
$3,1-5,19$ & 4,2 & 306 & 16 \\
$5,2-7,29$ & 6,3 & 181 & 16 \\
$7,3-9,39$ & 8,4 & 62 & 15 \\
$9,4-11,49$ & 10,5 & 19 & 14 \\
$11,5-13,60$ & 12,6 & 5 & 5 \\
\hline Total & & 665 & 80 \\
\hline
\end{tabular}

\section{Obtenção dos dados}

Em cada árvore abatida, foram medidas as alturas de cada um dos fustes $\left(\mathrm{H}_{\mathrm{f}}\right)$ e a altura total da árvore $\left(\mathrm{H}_{\mathrm{t}}\right)$, que correspondia à altura do fuste principal (fuste mais alto da árvore). A cubagem foi realizada através do método de Smalian, sendo considerado como uma árvore individual cada um dos fustes originados abaixo de 1,3 m, conforme Araújo et al. (2004).

Em cada fuste, foram medidas as circunferências com casca nas posições $0,1 \mathrm{~m}, 0,3 \mathrm{~m}, 0,7 \mathrm{~m}, 1,3 \mathrm{~m}, 2 \mathrm{~m}$ e 2,8 $m$ do nível do solo e a partir desse ponto as seções foram medidas em intervalos de $1 \mathrm{~m}$. Após a medição da última seção, obteve-se também o comprimento da ponta.
As medidas de circunferência e altura das árvores e fustes foram obtidas com auxílio de fita métrica e trena, respectivamente. Após a transformação das circunferências para diâmetros, foram calculados os volumes por seção. $\mathrm{O}$ volume total de cada fuste $\left(\mathrm{V}_{\mathrm{f}}\right)$ foi obtido pelo somatório do volume de todas as seções mais o volume da ponta. Por sua vez, o volume total de cada árvore $\left(\mathrm{V}_{\mathrm{t}}\right)$ foi obtido pelo somatório dos volumes totais dos fustes.

A partir dos DAP's dos múltiplos fustes, quando presentes, calculou-se o diâmetro equivalente de cada árvore (DEq), conforme Silva (2005). O DEq é dado pela expressão (1), a qual pressupõe que a área transversal (g) a 1,30 m de uma árvore (j) com diversos fustes (i) é produzida pela soma das áreas transversais individuais de cada fuste.

$$
D E q_{j}=\sqrt{D A P_{1}^{2}+D A P_{2}^{2}+\cdots+D A P_{n}^{2}}
$$

Onde: $\mathrm{DEq}_{\mathrm{j}}=$ diâmetro equivalente da j-ésima árvore na altura de 1,30 , em $\mathrm{cm}(\mathrm{j}=1,2, \ldots \mathrm{m}) ; \mathrm{DAP}_{\mathrm{i}}=$ diâmetro a 1,30 $\mathrm{m}$ do i-ésimo fuste, em $\mathrm{cm}$ ( $\mathrm{i}=$ fuste; $\mathrm{i}=1,2, \ldots \mathrm{n})$.

Assim, foram obtidos seis conjuntos de variáveis: $\mathrm{H}_{\mathrm{f}}, \mathrm{H}_{\mathrm{t}}, \mathrm{DAP}, \mathrm{DEq}, \mathrm{V}_{\mathrm{f}} \mathrm{e} \mathrm{V}_{\mathrm{t}}$, que foram combinadas para o ajuste dos modelos volumétricos.

\section{Métodos para predição do volume}

Foram avaliados três métodos comumente utilizados para estimativa do volume de madeira: fator de forma, quociente de forma e modelos volumétricos.

O fator de forma $(f)$ é definido pela razão entre o volume real da árvore e o volume do cilindro com diâmetro na altura de $1,30 \mathrm{~m}$ e altura total (Soares et al., 2006). O volume real da árvore foi determinado pela soma dos volumes individuais dos múltiplos fustes, enquanto o volume cilíndrico da árvore foi definido pela fórmula (2), que também considera o conjunto de fustes.

$$
\mathrm{Vcil}_{j}=\frac{\pi}{40.000} \cdot\left(D E q_{j}^{2}\right) \cdot H_{j}
$$

onde: $\mathrm{Vcil}_{\mathrm{j}}=$ volume cilíndrico da j-ésima árvore, $\mathrm{em} \mathrm{m}^{3}(\mathrm{j}$ $=1,2, \ldots \mathrm{m}) ; \mathrm{DEq}_{\mathrm{j}}=$ diâmetro equivalente da j-ésima árvore na altura de 1,30 , em $\mathrm{cm}(\mathrm{j}=1,2, \ldots \mathrm{m})$.

Para determinação do quociente de forma $(Q)$, definido pela razão entre diâmetros, adotou-se o quociente de Schiffel (Soares et al., 2006). Considerando a ocorrência de múltiplos fustes em uma árvore, o $Q$ foi calculado de acordo com a fórmula (3). 


$$
Q=\frac{D E q^{1 / 2} H_{j}}{D E q_{j}}
$$

onde: $\mathrm{DEq} 1 / 2 \mathrm{H}_{\mathrm{j}=}$ diâmetro equivalente da $\mathrm{j}$-ésima árvore na metade da altura total da árvore, em $\mathrm{cm}(\mathrm{j}=1,2, \ldots \mathrm{m})$, calculado a partir dos diâmetros medidos na posição correspondente a metade da altura de cada fuste durante a cubagem; $\mathrm{DEq}_{\mathrm{j}}=$ diâmetro equivalente da j-ésima árvore na altura de 1,30 , em $\mathrm{cm}(\mathrm{j}=1,2, \ldots \mathrm{m})$.

Os volumes individuais das árvores obtidos por meio do fator de forma e do quociente de forma foram calculados com base na média aritmética dos valores individuais de $f$ e de $Q$, a qual foi multiplicada pelo volume cilíndrico da árvore.

Os modelos volumétricos testados foram selecionados na literatura (Finger, 1992; Soares et al., 2006; Azevedo et al., 2011), totalizando um número de oito equações (Tabela 2), que tiveram duas opções para a variável dependente volume $\left(\mathrm{V}_{\mathrm{t}}\right.$ ou $\left.\mathrm{V}_{\mathrm{f}}\right)$ e para as variáveis independentes altura $\left(\mathrm{H}_{\mathrm{t}}\right.$ ou $\left.\mathrm{H}_{\mathrm{f}}\right)$ e/ou diâmetro (DEq ou DAP). Dessa forma, os modelos foram ajustados para os fustes $\left(\mathrm{V}_{\mathrm{f}, \mathrm{DAP}} \mathrm{e} \mathrm{H}_{\mathrm{f}}\right)$ e para as árvores, considerando o seu conjunto de fustes $\left(\mathrm{V}_{\mathrm{t}}\right.$, DEq e $\left.\mathrm{H}_{\mathrm{t}}\right)$. $\mathrm{O}$ ajuste de equações para os fustes e para as árvores não teve caráter comparativo para apontar a melhor delas, mas para ter a opção de escolha, visto que no primeiro caso tem-se como variável independente o DAP dos fustes e no segundo o DEq da árvore.

\section{Seleção dos modelos matemáticos}

A escolha do modelo de regressão mais adequado para estimativa do volume foi baseada nas seguintes estatísticas:

(a) Coeficiente de determinação $\left(\mathrm{R}^{2}\right)$, que expressa quanto da variação total é explicada pela regressão. Os valores de $R^{2}$ foram ajustados para que fosse possível comparar os valores encontrados, já que os graus de liberdade da regressão diferem entre os modelos (Scolforo, 1993).

(b) Erro-padrão da estimativa na forma absoluta e percentual $\left(\mathrm{S}_{\mathrm{yx}}\right)$, que indica a precisão do ajuste de um modelo matemático, informando o quanto o modelo erra, em média, ao estimar a variável dependente (Machado et al., 2008). Quanto menor o valor de $S_{y x}$, melhor e mais precisa é a estimativa. Nos modelos em que a variável dependente sofreu algum tipo de transformação logarítmica, o erro padrão foi corrigido na escala original, para possibilitar a comparação com os modelos aritméticos. Além disso, multiplicou-se o volume estimado pelo fator de correção de Meyer, para então proceder o recálculo.

(d) Valor de F, que corresponde ao valor de F calculado na análise da variância. Esse também foi utilizado como um dos parâmetros estatísticos na determinação do melhor modelo matemático. Quanto maior o valor de $\mathrm{F}$, melhor o ajuste da equação.

Tabela 2. Modelos de relação volumétrica utilizados para estimar o volume de árvores individuais

\begin{tabular}{cll}
\hline Número & \multicolumn{1}{c}{ Modelo } & \multicolumn{1}{c}{ Autor* } \\
\hline 1 & $V=\beta_{0}+\beta_{1} D+\varepsilon$ & Equação da reta \\
2 & $\operatorname{Ln}(V)=\beta_{0}+\beta_{1} \operatorname{Ln}(D)+\beta_{2} \operatorname{Ln}(H)+\varepsilon$ & Schumacher-Hall logaritimizado \\
3 & $\operatorname{Ln}(V)=\beta_{0}+\beta_{1} \operatorname{Ln}\left(D^{2} H\right)+\varepsilon$ & Spurr \\
4 & $V=\beta_{0}+\beta_{1} D^{2}+\varepsilon$ & Koperzky-Gehrhardt \\
5 & $V=\beta_{0}+\beta_{1} D+\beta_{2} D^{2}+\varepsilon$ & Hohenald-Krenm \\
6 & $V=\beta_{0}+\beta_{1} \operatorname{Ln}(D)+\beta_{2} \operatorname{Ln}(H)+\varepsilon$ & Schumacher-Hall modificado \\
7 & $V=\beta_{0}+\beta_{1} D^{2}+\beta_{2} D^{2} H+\beta_{3} H+\varepsilon$ & Stoate \\
8 & $V=\beta_{0}+\beta_{1} D+\beta_{2} H+\varepsilon$ & Schumacher-Hall
\end{tabular}

$\mathrm{V}=$ volume da árvore $\left(\mathrm{V}_{\mathrm{t}}\right)$ ou volume do fuste $\left(\mathrm{V}_{\mathrm{f}}\right) ; \beta_{\mathrm{i}}=$ coeficientes dos modelos; $\mathrm{H}=$ altura total da árvore $\left(\mathrm{H}_{\mathrm{t}}\right)$ ou altura total do fuste $\left(\mathrm{H}_{\mathrm{f}}\right.$ ) em m; D = DAP (diâmetro com casca 1,30 m do solo, em cm) ou DEq (diâmetro equivalente com casca a 1,30 m do solo, em cm); Ln = Logaritmo neperiano; $\varepsilon=$ erro aleatório; *Modelos citados nas seguintes fontes: Finger (1992); Soares et al. (2006) e Azevedo et al. (2011). 
(e) Valor ponderado dos escores dos parâmetros estatísticos (VP), que considera todas as variáveis estatísticas anteriormente descritas. Por esse motivo, representa uma síntese dos resultados, facilitando o processo de seleção do melhor modelo matemático. $\mathrm{O}$ valor ponderado foi determinado atribuindo-se valores ou pesos aos parâmetros estatísticos. As estatísticas foram ordenadas de acordo com a sua eficiência, sendo atribuído peso 1 para a equação mais eficiente, dois para a segunda e assim sucessivamente (ranking), conforme adotado por Thomas et al. (2006). Após essa classificação individual, efetuou-se o somatório da pontuação para cada modelo matemático, sendo que as duas equações de volume que receberam as menores somas foram indicadas como mais adequadas para uso.

(f) Análise gráfica dos resíduos, onde os modelos selecionados com base nos valores de VP foram analisados quanto à distribuição gráfica dos resíduos para avaliar a ocorrência de tendência nas estimativas da variável dependente.

\section{Métodos para obtenção do volume}

A fim de verificar qual método apresenta maior eficiência na estimativa do volume total de cada árvore, tendo como referência o valor obtido pela cubagem rigorosa, foram comparados os resultados de volumes totais encontrados por meio do fator de forma, do quociente de forma e dos modelos volumétricos selecionados em função do melhor desempenho para a estimativa do volume da árvore e do fuste.

Os resultados de volume obtidos foram analisados separadamente, segundo um delineamento inteiramente casualizado com cinco tratamentos (cubagem rigorosa, fator de forma, quociente de forma, modelo de Stoate para a árvore e para o fuste) e 30 repetições. Adotou-se o teste $\mathrm{F}$ a 5\%. De forma complementar, para comparação de médias, utilizou-se o teste de Tukey a 5\% de significância.

\section{Resultados e discussão}

\section{Modelos para volume total da árvore}

Os modelos testados apresentaram $\mathrm{R}^{2}{ }_{\text {aj }}$ variando de 75,0 a $96,1 \%$ (Tabela 3). Apenas três modelos tiveram valor de $\mathrm{R}_{\text {aj }}^{2}$ inferior a $93 \%$, sugerindo bom desempenho estatístico da maior parte dos modelos pelo alto grau de ajuste da variável dependente em função das variáveis independentes. Os modelos 2 e 7 apresentaram os maiores valores para essa estatística, em torno de $96 \%$. Silva (2005), também utilizando DEq como variável independente em modelos volumétricos para o conjunto de espécies ocorrentes em áreas de Caatinga na Paraíba e no Rio Grande do Norte, encontrou $\mathrm{R}_{\text {aj }}^{2}$ de $99,4 \%$ para o modelo 2 (Schumacher-Hall logaritimizado).

$\mathrm{O}$ erro padrão da estimativa em percentual $\left(\mathrm{S}_{\mathrm{yx}} \%\right)$ ficou entre 11,6\% e 28,8\% (Tabela 3). Dos oito modelos empregados, cinco apresentaram valores de $\mathrm{S}_{\mathrm{yx}} \%$ inferiores a $15 \%$, com menor resultado encontrado para o modelo 7 (11,6\%), seguido dos modelos 3 e 5 (13,64\% e 13,99\%, respectivamente). A mesma tendência foi verificada para os valores referentes ao erro padrão na forma absoluta, indicando maior eficiência do modelo $7\left(0,005 \mathrm{~m}^{3}\right)$ e menor do modelo $6\left(0,131 \mathrm{~m}^{3}\right)$. Todos os modelos testados apresentaram valores significativos para o $\mathrm{F}$ calculado (Tabela 3). Os maiores resultados foram observados para os modelos 3 e 4 (655,4 e 446,1, respectivamente).

Tabela 3. Coeficientes e parâmetros estatísticos obtidos no ajuste dos modelos para estimativa do volume total com casca de árvores de Pterogyne nitens em plantio puro no Município de Vitória da Conquista, BA.

\begin{tabular}{ccccccccccc}
\hline Modelo & $\boldsymbol{\beta}_{\boldsymbol{0}}$ & $\boldsymbol{\beta}_{\boldsymbol{1}}$ & $\boldsymbol{\beta}_{\mathbf{2}}$ & $\boldsymbol{\beta}_{\mathbf{3}}$ & $\mathbf{R}_{\mathrm{aj}}{ }_{\mathrm{j}}$ & $\mathbf{S}_{\mathbf{y x}}$ & $\mathbf{S}_{\mathbf{y x}} \boldsymbol{\%}$ & $\mathbf{F}$ & $\mathbf{V P}$ \\
\hline 1 & $-0,03013$ & 0,00681 & - & - & 88,07 & 0,00920 & 19,97 & 215,07 & 27 \\
2 & $-7,70301$ & 1,58387 & 0,35203 & - & 96,09 & 0,00646 & 14,31 & 357,35 & 13 \\
3 & $-7,89077$ & 0,68919 & - & - & 95,76 & 0,00614 & 13,64 & 655,40 & 8 \\
4 & 0,00047 & 0,00033 & - & - & 93,88 & 0,00651 & 14,30 & 446,10 & 16 \\
5 & 0,01316 & $-0,00264$ & 0,00045 & - & 94,15 & 0,00640 & 13,99 & 234,28 & 14 \\
6 & $-0,09537$ & 0,05470 & 0,00645 & - & 75,10 & 0,01310 & 28,85 & 44,73 & 32 \\
7 & 0,00110 & 0,00010 & 0,00002 & 0,00081 & 95,98 & 0,00530 & 11,59 & 231,71 & 9 \\
8 & $-0,03803$ & 0,00594 & 0,00234 & - & 88,44 & 0,00900 & 19,66 & 111,90 & 25 \\
\hline
\end{tabular}

$\beta i$ = coeficientes dos modelos; $R^{2}$ = coeficiente de determinação ajustado; Syx = erro-padrão da estimativa; Syx $\%$ = erro-padrão da estimativa em percentual; $\mathrm{F}=$ valor de $\mathrm{F}$ de análise de variância; $\mathrm{VP}=$ valor ponderado dos escores. 
De acordo com o valor ponderado (VP) (Tabela 3 ), os modelos 7 (Stoate) e 3 (Spurr) apresentaram melhor desempenho. Souza (2012), ajustando modelos volumétricos para espécies florestais da Caatinga em função do diâmetro equivalente (DEq), também verificou que o modelo de Spurr logaritimizado (modelo 3) apresentou resultados satisfatórios para a estimativa do volume de madeira, com valores de $\mathrm{R}^{2}{ }_{\text {aj }}$ e erro padrão da estimativa iguais a 96,3 e $2,6 \%$, respectivamente.

(A)

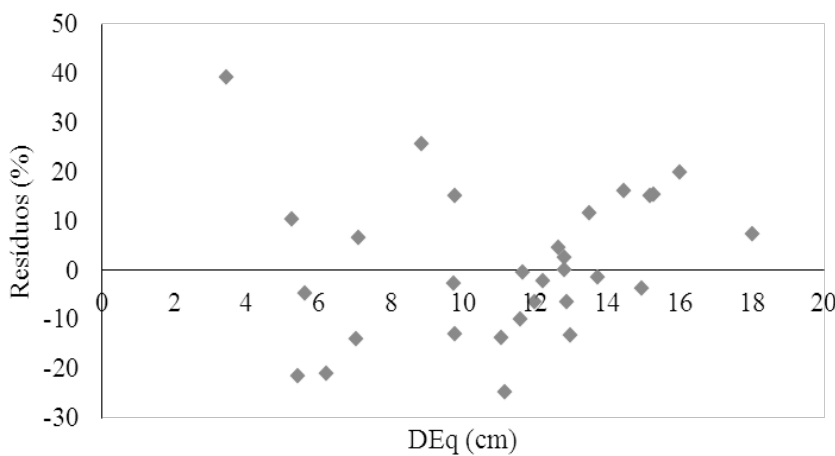

A dispersão gráfica dos resíduos em relação à média (Figura 1) demonstrou distribuição mais uniforme do modelo de Stoate em relação ao modelo de Spurr, o que sugere que este primeiro modelo seria mais indicado para estimar o volume total da árvore com casca em função das variáveis independentes DEq e altura total. Tonini \& Melo (2009), avaliando equações volumétricas para espécies florestais nativas no Município do Cantá (RR), também observaram melhores resultados do modelo de Stoate para a estimativa do volume total com casca de árvores de visgueiro (Parkia pendula Willd. Benth. ex Walp.).

(B)

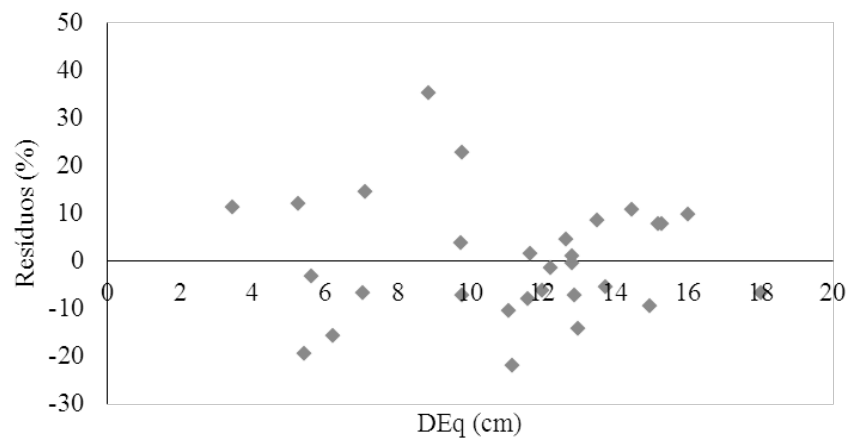

Figura 1. Distribuição dos resíduos dos modelos volumétricos de Spurr (A) e de Stoate (B) em função do diâmetro equivalente (DEq) de árvores de Pterogyne nitens em plantio puro no Município de Vitória da Conquista, BA.

\section{Modelos para volume do fuste}

Os resultados dos ajustes das equações testadas para a estimativa do volume do fuste com casca $\left(\mathrm{V}_{\mathrm{f}}\right)$, partindo dos dados da cubagem rigorosa dos fustes individualmente, em função das variáveis independentes (DAP ou DAP e $\mathrm{H}_{\mathrm{f}}$ ) estão apresentados na Tabela 4. Os modelos testados apresentaram $\mathrm{R}_{\text {aj }}^{2}$ variando de 73,6 a 91,4\%. Os valores observados para este parâmetro revelam que quatro dos modelos testados apresentaram melhor desempenho estatístico (modelos 2, 4, 5 e 7), com valores acima de $90 \%$, o que demonstra que o ajuste de descrição da variável dependente pelas variáveis independentes foi adequado.

Tabela 4. Coeficientes e parâmetros estatísticos obtidos no ajuste dos modelos para estimativa do volume com casca de fustes de Pterogyne nitens em plantio puro no Município de Vitória da Conquista, BA.

\begin{tabular}{cccccccccc}
\hline Modelo & $\boldsymbol{\beta}_{\boldsymbol{0}}$ & $\boldsymbol{\beta}_{\boldsymbol{1}}$ & $\boldsymbol{\beta}_{\boldsymbol{2}}$ & $\boldsymbol{\beta}_{\boldsymbol{3}}$ & $\mathbf{R}_{\mathrm{aj}}^{\mathbf{2}} \mathbf{\%}$ & $\mathbf{S}_{\mathbf{y x}}$ & $\mathbf{S}_{\mathbf{y x}} \boldsymbol{\%}$ & $\mathbf{F}$ & $\mathbf{V P}$ \\
\hline 1 & $-0,00948$ & 0,00410 & - & - & 85,73 & 0,00504 & 29,88 & 481,97 & 20 \\
2 & $-7,72692$ & 1,589967 & 0,271686 & - & 90,71 & 0,00427 & 24,84 & 369,17 & 17 \\
3 & $-7,87645$ & 0,63741 & - & - & 85,62 & 0,00600 & 35,59 & 477,50 & 24 \\
4 & 0,00057 & 0,00032 & - & - & 90,82 & 0,00404 & 23,96 & 793,06 & 7 \\
5 & 0,00172 & $-0,00042$ & 0,00036 & - & 90,74 & 0,00406 & 24,07 & 393,17 & 13 \\
6 & $-0,01706$ & 0,02288 & $-0,00312$ & - & 73,63 & 0,00685 & 40,63 & 112,72 & 31 \\
7 & 0,00057 & 0,00032 & $3,44 \mathrm{E}-09$ & $1,42 \mathrm{E}-06$ & 91,48 & 0,00389 & 23,09 & 269,54 & 8 \\
8 & $-0,00962$ & 0,00426 & $-0,00014$ & - & 86,45 & 0,00491 & 29,12 & 256,24 & 21 \\
\hline
\end{tabular}

$\beta i$ = coeficientes dos modelos; $R^{2}=$ coeficiente de determinação ajustado; Syx = erro-padrão da estimativa; Syx $\%$ = erro-padrão da estimativa em percentual; $\mathrm{F}=$ valor de $\mathrm{F}$ de análise de variância; $\mathrm{VP}=$ valor ponderado dos escores. 
Com relação ao $\mathrm{S}_{\mathrm{yx}}$, os modelos de Schumacher-Hall logaritimizado, Koperzky-Gehrhardt, Hohenald-Krenm e de Stoate apresentaram menor valor $\left(0,004 \mathrm{~m}^{3}\right)$, sugerindo maior eficiência.

Os valores de $\mathrm{S}_{\mathrm{yx}} \%$ estiveram entre $23,1 \%$ (modelo 7) e 40,6\% (modelo 6) (Tabela 4). Uma das possíveis causas desses altos valores pode ser a grande heterogeneidade do povoamento, que propiciou maior magnitude dos erros associados aos modelos testados. Os menores números observados nos modelos 7, 4 e 2 (média de $24 \%$ ) representam menor variabilidade dos dados estimados a partir desses modelos.

De acordo com os valores de F, destacaram-se os modelos $4(793,0), 1(482,0)$ e $3(477,5)$. No entanto, considerando os valores ponderados dos escores estatísticos (VP), os melhores resultados foram observados para os modelos 4 (Koperzky-Gehrhardt) e 7 (Stoate) (Tabela 4).

De acordo com Ahrens (1982), mesmo que os índices de ajuste $\mathrm{R}^{2}$ aj e de precisão $\mathrm{Syx} \%$ sejam julgados satisfatórios, torna-se relevante avaliar a distribuição dos resíduos, pois é esta que vai indicar se a estimativa é boa ao longo de toda a linha de regressão, se não há tendências em nenhum de seus segmentos e se o modelo é apropriado para tal fim.

A distribuição gráfica dos resíduos dos dois modelos selecionados (modelos com menores valores de VP) para a estimativa do volume total do fuste, em função do DAP, está apresentada na Figura 2.
(A)

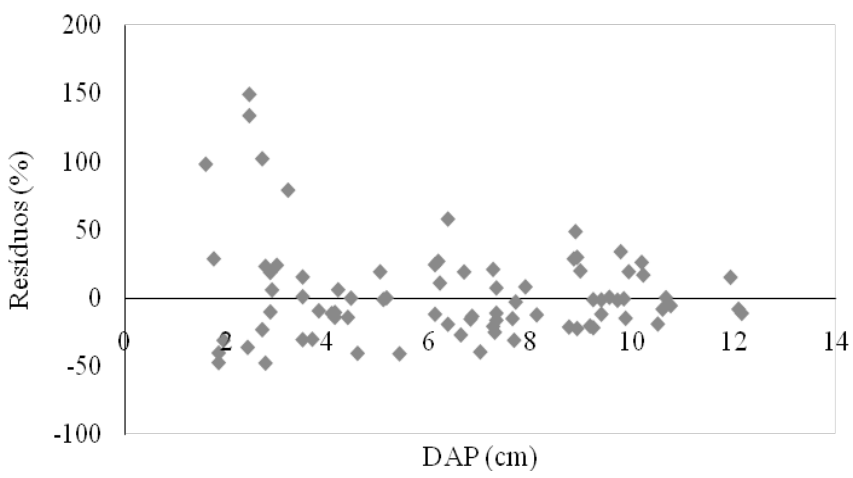

(B)

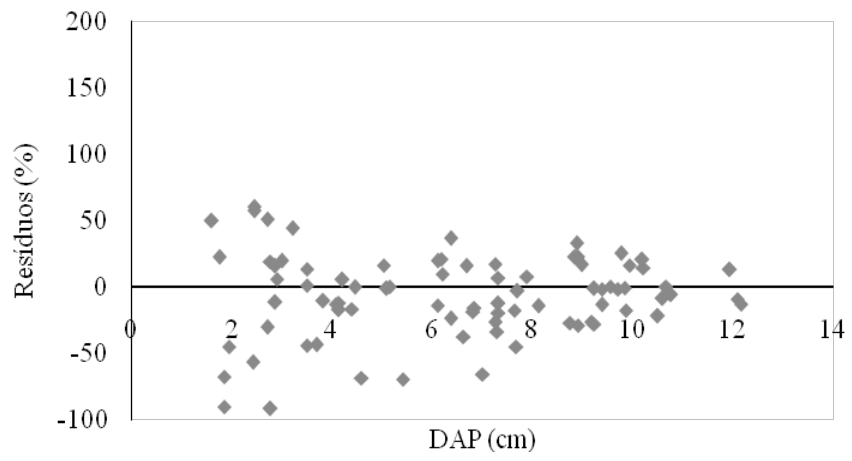

Figura 2. Distribuição dos resíduos dos modelos de Koperzky-Gehrhardt (A) e Stoate (B) em função do diâmetro a 1,3 m de altura (DAP) de fustes de Pterogyne nitens, em plantio puro, no Município de Vitória da Conquista, BA.

Os resíduos máximos positivos e mínimos negativos foram de 149,75 e 47,91\% (Koperzky-Gehrhardt) e 60,03 e 90,07\% (Stoate), respectivamente. Adistribuição gráfica dos resíduos não demonstrou tendências nítidas em nenhum dos dois modelos selecionados (Figura 2). Embora o modelo de Stoate tenha propiciado resíduos mais uniformemente distribuídos em relação à média, o modelo de Koperzky-Gehrhardt também apresentou bom desempenho na distribuição, demonstrando apenas uma leve tendência de subestimação para indivíduos com menor DAP (entre 2 e $3 \mathrm{~cm}$ ). Esse resultado sugere que ambos os modelos são apropriados para estimar o volume do fuste com casca da espécie estudada.

\section{Métodos para estimativa do volume total}

Os valores médios de fator de forma $(f)$ e quociente de forma $(\mathrm{Q})$ das árvores de madeira-nova foram de 0,6 e 0,7 , respectivamente. Esses valores são esperados para plantios jovens, corroborando com resultados já publicados na literatura que indicam que, em geral, em árvores mais jovens o fator de forma apresenta uma relação mais próxima da forma cilíndrica, enquanto em indivíduos mais velhos a conicidade é maior (Drescher et al., 2001; Figueiredo et al., 2005).

Azevedo et al. (2011), estudando métodos de estimativa de volume de Eucalyptus urophylla com cinco anos, na mesma região de estudo do presente trabalho, 
encontraram valor médio de fator de forma de 0,49 e de quociente de forma de 0,64 . Resultado semelhante foi observado por Tonini et al. (2005), estudando a volumetria de espécies nativas, onde obtiveram forma de 0,5 para Andiroba (Carapa guianensis Aubl.) e de 0,4 para Ipê roxo (Tabebuia avellanedae Lorentz ex Griseb.).

Os volumes estimados por meio do fator de forma e quociente de forma apresentaram valores estatisticamente iguais entre si e superiores ao volume obtido com a cubagem, demonstrando tendência, tanto do fator quanto do quociente de forma, de superestimar o volume. Por outro lado, os volumes estimados pelas equações ajustadas, tanto em função de DEq quanto em função do DAP, não apresentaram diferenças significativas em relação ao volume da cubagem, evidenciando o maior desempenho do modelo selecionado (Stoate) na estimação do volume da espécie estudada. Tonini et al. (2005) também verificou maior eficiência do modelo de Stoate como método de estimativa de volume com casca de Jatobá (Hymenaea courbaril L.).

Tabela 6. Volume real e volumes estimadospor meio do fator de forma médio, quociente de forma médio e modelo de Stoate, em função do DEq e em função do DAP, para Pterogyne nitens, em plantio puro, no município de Vitória da Conquista, BA.

\begin{tabular}{ccccc}
\hline $\mathbf{V ~ r e a l}^{(1)}$ & $\mathbf{V}(\boldsymbol{f})$ & $\mathbf{V}(\boldsymbol{Q})$ & $\mathbf{V}\left(\mathbf{D E q}, \mathbf{H}_{\mathbf{t}}\right)$ & $\mathbf{V}\left(\mathbf{D A P}, \mathbf{H}_{\mathbf{f}}\right)$ \\
\hline $0,04553 \mathrm{~b}$ & $0,05668 \mathrm{a}$ & $0,05746 \mathrm{a}$ & $0,04467 \mathrm{~b}$ & $0,04553 \mathrm{~b}$ \\
\hline
\end{tabular}

(1) Vreal= volume médio da cubagem rigorosa; $\mathrm{V}(\mathrm{f})=$ volume médio estimado em função do fator de forma; $V(Q)=$ volume médio estimado em função do quociente de forma; $\mathrm{V}(\mathrm{DEq})=$ volume médio estimado por meio do modelo de Stoate ajustado em função de DEq e altura total da árvore; V (DAP)= volume médio estimado por meio do modelo de Stoate ajustado em função do DAP e altura do fuste; Médias seguidas pela mesma minúscula não diferem entre si pelo teste de Tukey a 5\% de significância.

\section{Conclusão}

Dentre os métodos utilizados para estimar o volume, o mais recomendado é o emprego de modelos volumétricos ajustados.

O modelo de Stoate apresentou melhor performance para estimar tanto o volume do fuste quanto o volume total da árvore com casca de Pterogyne nitens. Todavia, para o volume do fuste, o modelo de Koperzky-Gehrhardt também proporcionou estimativas semelhantes ao anterior. Nesse contexto, como a determinação da altura das árvores é uma operação onerosa e sujeita a erros, a utilização do modelo de Koperzky-Gehrhardt pode representar redução de tempo e custos das operações de inventário florestal, uma vez que esse modelo estima o volume em função apenas do DAP.

\section{Referências}

AHRENS, S. Importância da distribuição de resíduos de regressão na seleção de equações de volume. In: EMBRAPA. Unidade Regional de Pesquisa Florestal Centro-Sul (Curitiba).Contribuição da URPFCS ao 4. Congresso Florestal Brasileiro. Curitiba: EMBRAPAURPFCS, 1982. p. 7-25. (EMBRAPA-URPFCS. Documentos, 10). Edição dos Resumos do $4^{\circ}$ Congresso Florestal Brasileiro, Belo Horizonte, 1982.

ALMEIDA, R. C.; CABACINHA, C. D.; ROCHA, T. B.; PAULA, A. de. Volumetria de Eucalyptus urophylla S.T. Blake pelo método geométrico no Planalto de Conquista, Bahia. Revista Caatinga, Mossoró, v. 24, n. 1, p. 65-75, 2011.

ARAÚJO, L. V. C.; LEITE, J. A. N.; PAES, J. B. Estimativa da produção de biomassa de um povoamento de jurema preta (Mimosa tenuiflora Willd. Poiret.) com cinco anos e idade. Biomassa \& Energia, Viçosa, MG, v. 1, n. 4, p. 347-352, 2004.

AZEVEDo, G. B.; SOUSA, G. T. de O.; BARreto, P. A. B.; CONCEIÇÃO JUNIOR, V. Estimativas volumétricas em povoamentos de eucalipto sob regime de alto fuste e talhadia no Sudoeste da Bahia. Pesquisa Florestal Brasileira, Colombo, v. 31, n. 68, p. 309-318, 2011. DOI: 10.4336/2011.pfb.31.68.309

CAMPOS, J. C. C.; LEITE, H. G. Mensuração florestal: perguntas e respostas. 2. ed. Viçosa, MG: Ed da UFV, 2006. 470 p.

CARVALHO, P. E. R. Espécies florestais brasileiras: recomendações silviculturais, potencialidas e uso da madeira. Colombo: EMBRAPA/ CNPF; Brasília, DF: EMBRAPA-SPI, 1994. 640 p.

DRESCHER, R.; SCHNEIDER, P. R.; FINGER, C. A. G.; QUEIROZ, F. L. C. Fator de forma artificial de Pinus elliottii Engelm para a região da Serra do Sudeste do estado do Rio Grande do Sul. Ciência Rural, Santa Maria, RS, v. 31, n. 1, 2001.

FERRARI, M. P.; FERREIRA, C. A.; SILVA, H. D. da; TREVISAN, R. Prognose do crescimento volumétrico individual de árvores de Eucalyptus, em povoamentos na região Centro Sul. Boletim Pesquisa Florestal, Colombo, n. 51, p. 5-16, jul./dez. 2005.

FIGUEIREDO, E. O.; SCOLFORO, J. R. S.; OLIVEIRA, A. D. de. Estimativa do percentual de casca e do fator de forma em povoamentos jovens de teca (Tectona grandis L.F.). Rio Branco, AC: Embrapa Acre, 2005. 5 p. (Embrapa Acre. Comunicado técnico, 165).

FINGER, C.A.G. Fundamentos de Biometria Florestal. Santa Maria, RS: UFSM/CEPEF/FATEC, 1992. 269 p.

FRANCO, A. A.; DIAS, L. E.; FARIA, S. M. de; CAMPELLO, E. F. C.; SILVA, E. M. R. da . Uso de leguminosas florestais noduladas e micorrizadas como agentes de recuperação e manutenção da vida no solo: um modelo tecnológico. Oecologia Brasiliensis, Rio de Janeiro, RJ, v. 1, p. 459-467, 1995.

LAMPRECHT, H. Silvicultura nos trópicos: ecossistemas florestais e respectivas espécies arbóreas: possibilidades e métodos de aproveitamento sustentado. Eschborn: GTZ, 2000. 343 p.

LORENZI, H. Árvores brasileiras: manual de cultivo de plantas arbóreas nativas do Brasil. Nova Odessa: Plantarum, 2002. v. 1. 
MACHADO, S. A.; NASCIMENTO, R. G. M.; AUGUSTYNCZIK, A. L. D.; SILVA, L. C. R. da; FIGURA, M. A.; PEREIRA, E. M.; TÉO, S. J. Comportamento da relação hipsométrica de Araucaria angustifolia no capão da Engenharia Florestal da UFPR. Pesquisa Florestal Brasileira, Colombo, n. 56, p. 5-16, 2008.

MACHADO, S. A.; URBANO, E.; CONCEIÇÃO, M. B. Comparação de métodos de estimativa de volume para Pinus oocarpa em diferentes idades e diferentes regimes de desbastes. Boletim Pesquisa Florestal, Colombo, n. 50, p. 81-98, 2005.

MELO, L. C.; BARRETO, P. A. B.; OliveIRA, F. G. R. B. de; NOVAES, A. B. de. Estimativas volumétricas em povoamento de Pinus caribaea var. hondurensis no sudoeste da Bahia. Pesquisa Florestal Brasileira, Colombo, v. 33, n. 76, p. 379-386, 2013. DOI: http://dx.doi.org/10.4336/2013.pfb.33.76.459

MORAES NETO, S. P. Estimativas de volume de Pinus tecunumanii no Cerrado do Distrito Federal. Planaltina, DF: Embrapa Cerrados, 2009. 16 p. (Embrapa Cerrados. Boletim de Pesquisa e Desenvolvimento, 231).

OLIVEIRA, M. L. R.; LEITE, H. G.; GARCIA, S. L. R.; CAMPOS, J. C. C.; SOARES, C. P. B.; SANTANA, R. C. Estimação do volume de árvores de clones de eucalipto pelo método da similaridade de perfis. Revista Árvore, Viçosa, MG, v. 33, n. 1, 2009.

PELISSARI, A. L.; LANSSANOVA, L. R.; DRESCHER, R. Modelos volumétricos para Pinus tropicais, em povoamento homogêneo, no Estado de Rondônia. Pesquisa Florestal Brasileira, Colombo, v. 31, n. 67, p. 173-181, 2011.

RIOS, P. D'A.; MORI, F. A. Estimativa de idade das árvores, massa específica e cubagem da casca de Kielmeyera coriacea Mart. Scientia Florestalis, Piracicaba, v. 40, n. 93, p. 85-93, 2002.

SANTOS, H. G. dos; JACOMINE, P. K. T.; ANJOS, L. H. C. dos; OLIVEIRA, V. A. de; OLIVEIRA, J. B. de; COELHO, M. R.; LUMBRERAS, J. F.; CUNHA, T. J. F. (Ed.). Sistema brasileiro de classificação de solos. 2. ed. Rio de Janeiro: Embrapa Solos, 2006. 306 p.

SCOLFORO, J. R. Mensuração Florestal 3: relações quantitativas em volume, peso e a relação hipsométrica. Lavras: ESAL/FAEPE, 1993. $292 \mathrm{p}$.

SCOLFORO, J. R. S.; FIGUEIREDO FILHO, A. Biometria florestal: medição e volumetria de árvores. Lavras: UFLA/FAEPE, $1998.310 \mathrm{p}$.

SILVA, J. A. Fitossociologia e relações alométricas em caatinga nos estados da Paraíba e Rio Grande do Norte. 2005. 93 f. Tese (Doutorado em Ciência Florestal) - Universidade Federal de Viçosa, Viçosa, MG.
SOARES, C. P. B.; PAUlA NETO, F. de; SOUZA, A. L. Dendrometria e inventário florestal. Viçosa, MG: Universidade Federal de Viçosa, 2006. 276 p.

SOARES, C. P. B.; SILVA, G. F.; MARTINS, F. B. Influence of section lengths on volume determination in Eucalyptus trees. Cerne, Lavras, v. 16, n. 2, p. 155-162, 2010.

SOUSA, G. T. O.; AZEVEDO, G. B.; BARRETO, P. A. B.; CONCEIÇÃO JÚNIOR, V. Relações hipsométricas para Eucalyptus urophylla conduzidos sob regime de alto fuste e talhadia no Sudoeste da Bahia. Scientia Plena, Aracaju, v. 9, n. 4, p. 1-7, 2013.

SOUZA, P. F. Estudos fitossociológicos e dendrométricos em um fragmento de caatinga, São José de Espinharas - PB. 2012. 97 f. Dissertação (Mestrado em Ciências Florestais) - Universidade Federal de Campina Grande, Patos, PB.

THOMAS, C.; ANDRADE, C. M.; SCHNEIDER, P. R.; FINGER, C. A. J. Comparação de equações volumétricas ajustadas com dados de cubagem e análise de tronco. Ciência Florestal, Santa Maria, RS, v. 16, n. 3, p. 319-327, 2006.

TONINI, H.; ARCO-VERDE, M. F.; SÁ, S. P. P. Dendrometria de espécies nativas em plantios homogêneos no Estado de Roraima Andiroba (Carapa guianensis Aubl), Castanha-do-Brasil (Bertholletia excelsa Bonpl.), Ipê-roxo (Tabebuia avellanedae Lorentz ex Griseb) e Jatobá (Hymenaea courbaril L.). Acta Amazonica, Manaus, v. 35, n. 3, p. 353-362, 2005.

TONINI, H.; MELO, S. R. Equações volumétricas para espécies nativas e exóticas no município do Cantá, RR. Boa Vista: Embrapa Roraima, 2009. 17 p. (Embrapa Roraima, Boletim de Pesquisa e Desenvolvimentos, 16).

VASCONCELOS, F. Manejo para produção de lenha: MMA e SFB ajudarão agricultores e indústrias da região da caatinga a obter matriz energética de forma ambientalmente sustentável. InforMMA Notícias, 13 jul. 2012. Disponível em: <http://www.mma.gov.br/ informma/item/8513-manejo-para-produ $\% \mathrm{C} 3 \% \mathrm{~A} 7 \% \mathrm{C} 3 \% \mathrm{~A} 30$-delenha>. Acesso em: 24 ago. 2012.

VEloso, H. P.; RANGEL FILHO, A. L. R.; LIMA, J. C. A. Classificação da vegetação brasileira adaptada a um sistema universal. Rio de Janeiro, RJ: IBGE, Departamento de Recursos Naturais e Estudos Ambientais, 1991. 124 p. 
